\title{
Dramatic changes to well-known places go unnoticed
}

Rosenbaum, R.S. ${ }^{12^{*}}$, Halilova, J.G. ${ }^{1}$, Agnihotri, S. ${ }^{1,3}$, D’Angelo, M.C. ${ }^{2}$, Winocur, G. ${ }^{2}$, Ryan, J.D. ${ }^{2,3}, \&$ Moscovitch, M. ${ }^{2,3}$

${ }^{1}$ York University, Toronto, ON, Canada; ${ }^{2}$ Rotman Research Institute, Baycrest, Toronto, ON, Canada; ${ }^{3}$ University of Toronto, ON, Canada 


\begin{abstract}
How well do we know our city? It turns out, much more poorly than we might imagine. We used declarative memory and eye-tracking techniques to examine people's ability to detect modifications of landmarks in Toronto locales with which they have had extensive experience. Participants were poor at identifying which scenes contained altered landmarks, whether the modification was to the landmarks' relative size, internal features, or surrounding context. To determine whether an indirect measure would prove more sensitive, we tracked eye movements during viewing. Changes in overall visual exploration, but not to specific regions of change, were related to participants' explicit endorsement of scenes as modified. These results support the contention that very familiar landmarks are strongly integrated within the spatial context in which they were first experienced, so that any changes that are consciously detected are at a global or coarse, but not local or fine-grained, level.
\end{abstract}

Keywords: spatial memory, remote memory, navigation, landmark recognition, eye tracking 
RUNNING HEAD: MEMORY FOR WELL-KNOWN PLACES

\section{Statement of Relevance}

Studies of newly experienced environments show that the geometry or configural relations are encoded rather than visual appearance details. How do we represent very familiar places that we experience often? Do time and experience allow us to represent environments in greater detail? We test the possibility that only schematic information or the basic configuration of landmarks is preserved in scenes that were acquired long ago. We show that individuals are unlikely to detect changes to the visual appearance of highly familiar and extensively experienced landmarks and scenes, whether tested directly by recognition or indirectly by eye movements; if they are detected, it is at a global or coarse level. The results seem counterintuitive: increased experience does not always lead to greater precision. These findings offer a unified view of schemas across verbal and spatial domains, and across recent and remote memory, with implications for hippocampal-neocortical interactions. 
Consider this photograph of a popular destination located in downtown Toronto (Figure 1). Imagine that you have visited this location countless times over many years. Would you be able to determine if it is an accurate depiction of the scene? Here we present data to show that we are notoriously poor at making this type of distinction.

The crucial ability of organisms to navigate their environment in search of basic needs, such as food and shelter, has long been thought to involve the maintenance of precise and accurate calculations of locales in relation to each other within an allocentric spatial representation. A map-like code, termed a 'cognitive map' (Epstein, Patai, Julian, \& Spiers, 2017; O'Keefe and Nadel, 1978), is derived from the operation of an intricate network of place cells in the hippocampus in the context of grid, border, boundary, and head-direction cells in the hippocampus and other navigation-relevant brain structures (Ekstrom, Spiers, Bohbot, \& Rosenbaum, 2018; Epstein et al., 2017; Hunsaker \& Kesner, 2018; Moser, Moser, \& McNaughton, 2017). A cognitive map signals an organism's location based on distal boundaries of the environment it is navigating, independent of the direction it is facing. It is viewed as a flexible counterpart to the less flexible body-centred representation of the organism in relation to local environmental features. Once formed, a cognitive map is believed to remain stable over time (Hainmueller \& Bartos, 2018; Lever et al., 2002; Quiroga et al., 2005). Less clear is the amount and type of detail that is initially encoded and retained within long-term representations of familiar environments. In the current study, we alter the visual properties of landmarks and their spatial locations to examine the nature of participants' long-term representations of their environment.

Findings from lesion and electrophysiological studies are consistent with the Cognitive Map Theory (CMT) in showing that the acquisition of newly encountered places requires the 
hippocampus (Guderian et al., 2015; Morris et al., 1982; O’Keefe \& Dostrovsky, 1971). However, they are also consistent with alternative theories that view the hippocampus as playing a role in declarative (explicit) memory encoding generally (Scoville \& Milner, 1957; Squire, Genzel, Wixted, \& Morris, 2015) or in coding the relational structure inherent in spatial representations (Eichenbaum, 2017; Ryan, Althoff, Whitlow, \& Cohen, 2000). Some have proposed that it is the geometric aspects of the environment that determine these relations (Burgess, 2008; Cheng \& Newcombe, 2005; Lee \& Spelke, 2010), while others have suggested that it is a configuration that includes landmarks in particular locations (Manns \& Eichenbaum, 2009; O'Keefe \& Nadel, 1978). What these theories do not consider are the nature of the landmarks themselves, and whether the internal features or simply the relations of one to the other are important.

Inconsistent with CMT are findings that amnesic patients with hippocampal damage can navigate and represent spatial relations contained in environments that were learned long before the onset of their amnesia (Rosenbaum et al., 2000; Teng \& Squire, 1999). More detailed aspects of these representations, however, such as the visual appearance of routes or perceptual features of landmarks, may be impoverished (Herdman et al., 2015; Maguire, Nannery, \& Spiers, 2006; Rosenbaum et al., 2000). One possibility is that spatial memories, like other types of declarative or relational memories, can change over time into a schematic or gist-like form (Rosenbaum et al., 2001; Moscovitch, Cabeza, Winocur, \& Nadel, 2016). This process may resemble the 'semanticization' of episodic memories (Cermak \& O’Connor, 1983), whereby memory traces of repeated events become integrated with pre-existing knowledge in neocortex, stripped of the specific contextual details that would allow for rich re-experiencing of the event (Moscovitch et al., 2016). All of these theories emphasize the relational component of landmarks with respect to 
one another over their precise or incidental visual properties. To our knowledge, there has been very little information as to whether this, in fact, is the case for well-known environments.

Before it is possible to distinguish among these theories, it is necessary to determine what type of information is retained about landmarks. Here we test the possibility that only schematic information or the basic configuration of landmarks is preserved in scenes that were acquired long ago. We examined whether individuals with experience navigating downtown Toronto could detect changes to the visual features and relative size and placement of well-known Toronto landmarks. Two methods of investigation were used: 1. declarative responses to scene alterations, and 2. tracking eye movements, which are sensitive to alterations even if participants are not aware of them (Hannula, Ryan, \& Warren, 2017). There is a growing body of research supporting eye movements as a valid assay of long-term memory representations of faces and scenes (Ryan, Shen, \& Liu, 2020). A combination of these methods can provide broader insight into the types of details maintained in spatial memory and accessibility of those details to conscious awareness.

If spatial memories of well-known environments learned in the remote past include rich, contextual details, then participants should perform well on behavioral tasks designed to detect modifications to landmarks that require detailed recollection. This should be expressed in eye movement behaviour, with the eyes directed to the specific region of change, similar to what is observed in studies of recently learned images (Ryan et al., 2000; Smith, Hopkins, \& Squire, 2006). If, however, well-known environments are represented in a schematic way, lacking rich contextual details, then participants should perform poorly in detecting changes. Similarly, any difference in eye movements between modified and unmodified images should be reflected as 
general changes in visual exploration and should not be specific to, or within the vicinity of, the modification.

\section{Methods}

\section{Participants}

A total of 85 healthy adults with experience navigating through the downtown Toronto area participated across 5 experiments. The number of participants and range in years of experience with downtown Toronto varied with each experiment as detailed below. The study was conducted with the approval of the York University and Baycrest Health Sciences Ethics Review Boards. Each participant provided informed consent and was financially compensated for the time that they contributed.

\section{Apparatus and Stimuli}

Images of well-known downtown Toronto landmarks were prepared using Adobe Photoshop 8.0. They were presented either in their true (i.e., unmodified) form or were modified according to four main types of changes: (1) details of landmark (alterations to façade, color, dimensions); (2) details of surrounding area (added or removed surrounding buildings); (3) transposition (placing a known landmark in another known, but incorrect, location); (4) unusual viewpoint (same as change (2), but with an unusual view of the landmark). Stimuli were presented one at a time on a 17-inch Dell M991 monitor for Experiments 1-4, and on a 19-inch Dell M991 monitor (resolution 1024x768) from a distance of 24 inches for Experiment 5.

\section{Procedure}

Five different experimental procedures were used to provide converging evidence:

\section{Experiment 1}


A group of 20 participants (15 females; age, mean=29.1 years, $S D=7.06$ years) were asked to identify whether the image presented was a true representation of the landmark, or if it had been modified according to the four conditions described above. If their response was that the image had been modified, they were asked to describe what type of modification was used. Twelve of the participants were presented with 86 images that consisted of 43 unmodified (original) images and 43 of the corresponding modified images. In an effort to ensure that participants' poor performance was not due to interference from viewing both the unmodified and modified versions of the same stimulus, each image was presented only once to 5 additional participants, either as unmodified or modified, with the two versions counterbalanced between participants. Yet another 3 participants were presented with a slightly larger set of 58 images (32 unmodified, 26 modified). Years lived in Toronto ranged from 0.5 to 25 years across the 3 versions of the task. No differences in performance were detected between the tasks, so the 3 groups of participants were combined into a single group of 20.

\section{Experiment 2}

A new group of 8 participants ( 7 female; age, mean=24, $S D=3.25$ ) read directions that led to specific locations in downtown Toronto. After the directions were read, an image from one of the following four conditions was presented to the first 6 participants: (1) true location and true landmark; (2) true location but a modified version of the true landmark; (3) true location and true landmark, but a modified version of the surrounding area, and (4) different location and landmark. They were then asked whether the image was an accurate representation of the location one would arrive at having followed the given directions. Forty trials were presented that included ten of each condition. Because participants performed at ceiling in condition (4), that condition was excluded from analysis. An additional 2 participants were tested on only the 
first 3 conditions, with 10 trials per condition, for a total of 30 trials. Years experience navigating in Toronto ranging from 2 to 20 years.

\section{Experiment 3}

The primary question in the first two experiments was whether participants could detect if an image was modified when presented one at a time. We wanted to determine if performance would improve if each modified image was presented together with its unmodified counterpart, and if there were differences in performance depending on type of modification made. An additional 9 participants ( 7 female; age, mean=24.22, $S D=1.79$ ) were presented with the initial set of 43 true and 43 modified images from Experiment 1, with the unmodified image and its corresponding modified image presented side by side in a forced choice format. Participants were asked to identify which of the two images was a true representation. Years living in Toronto ranged from 1 to 26 years.

\section{Experiment 4}

To determine any age differences in the ability to detect if a well-known landmark had been modified, we tested a separate sample of 24 participants: 12 young adults (half female), with a mean age of 22.17 years $(S D=1.27)$ and with 12 to 25 years living in Toronto, and 12 older adults (half female) with a mean age of 64.83 years $(S D=8.92)$ and with 24 to 76 years living in Toronto. All participants rated their confidence navigating in familiar places as a 4 or 5 on a 5-point Likert scale. Twenty-four landmarks were selected from the set of images from Experiment 1. For each condition, there was a set of 8 unique landmarks. Each participant viewed half of the landmarks in their modified form and half in their true form. Half of each set was presented in its modified form for half the participants and in its original form for the other half of the participants. Each landmark was modified in one of the following ways: (1) a 
characteristic feature of the landmark was removed; (2) the size of the landmark was changed in relation to its surroundings; and (3) the landmark was transposed to another familiar location.

\section{Experiment 5}

A new group of 24 healthy young adults (half female) with a mean age of 24.83 years $(S D=3.81)$ was tested to determine if the results from Experiment 4 could be replicated and to assess eye movements. Participants' number of years of living in Toronto ranged from 1.5 years to 30 years. All participants rated their confidence navigating in familiar places as a 4 or 5 on a 5-point Likert scale. Twenty-two true images and modified images (feature change, size change, or transposition to a new location) of downtown Toronto landmarks were included. The images were based on those from Experiment 1 but had been retaken to ensure that they depicted recent changes to the city made within the 2 years since the behavioural experiments had taken place. After all the images were passively viewed during the eye tracking component of the experiment (described below), participants were informed that some of the images may have been modified and of the types of possible modifications. They were then asked to view each image without the eye-tracking equipment and verbally indicate if they were aware of what landmark was represented in the image, whether the image had been modified, and, if so, in what way. These responses were recorded during testing to determine accuracy of identification of modifications.

Eye tracking. An SR Research Ltd. EyeLink II head-mounted system was used to collect eye movement data at a rate of $500 \mathrm{~Hz}$, with a temporal resolution of $2 \mathrm{~ms}$ and a spatial resolution of 0.1 degrees. Prior to the start of the experiment, participants were presented with a 9-point calibration which was repeated if there was an error at any point greater than 1 degree. Drift corrections were performed as necessary at the beginning of each trial. For each trial, the image was presented for $7 \mathrm{~s}$. Participants were instructed to view the image freely and were not 
told that any of the images had been modified. A counterbalanced design was used such that each image was viewed equally as true or modified across participants (i.e., 12 participants viewed an image in its modified form and 12 viewed the same image unchanged).

\section{Data Analysis}

The analysis followed guidelines for analyzing signal detection data using mixed models, as this approach has been found to be superior to the traditional signal detection approach (Wright, Horry, \& Skagerberg, 2009). The analyses were conducted using logistic mixed models in R package lme4 (Bates, Maechler, \& Bolker, 2012). In Experiments 1, 2, 4 and 5, the odds of participants responding that the image was modified was used as the outcome variable to assess their ability to discriminate between modified and unmodified trials.

For the eye tracking portion of Experiment 5, changes in viewing that may have reflected some aspect of spatial memory for relations among elements of the landmark and other scene elements depicted in the image were determined by calculating differences in eye movements between manipulated images and true images. In addition, interest areas for the region of the image in which the manipulation would appear were created using SR Research Data Viewer software. In line with previous research reporting increased viewing of objects in a scene that have undergone a change from a prior viewing (e.g., Ryan et al., 2000), we assessed eye movements within the interest area in which the manipulation would occur.

\section{Results}

\section{Experiment 1}

A logistic mixed model was constructed with years lived in Toronto and display type (modified, unmodified) as fixed factors, stimulus image as a random intercept, display type as a random slope, and response (modified, unmodified) as the outcome. Years of experience 
navigating in Toronto was associated with participants being significantly more likely to respond that the presented image was modified, $b=-0.02, S E=0.007, Z=3.32, p<.001, \mathrm{OR}=1.02$, 95\% CI [1.01, 1.04]. Because number of years lived in Toronto may be associated with a response bias, this variable was controlled for in subsequent experiments. Controlling for years lived in Toronto, viewing a modified image significantly improved the odds of participants responding that the image was modified, compared to when they viewed an unmodified image (see Table 1 and Figure 2a). Considering how marked the change was, detectability was not high.

\section{Experiment 2}

A logistic mixed model was constructed with display type (modified, unmodified) and years lived in Toronto as fixed factors, subject and image as random intercepts, and responding whether the image is modified or not as the outcome variable. Controlling for years lived in Toronto, viewing a modified image compared to an unmodified image increased the odds of participants responding that the image was modified (see Table 1 and Figure $2 b$ ). However, the increase was not statistically significant.

\section{Experiment 3}

A logistic mixed model was constructed with subject as a random intercept, and familiarity and modification type as fixed factors. A likelihood ratio test showed that adding modification type (i.e., landmark details $[M=0.63, S D=0.48]$, surrounding area details $[M=$ $0.67,0.47]$, transposition $[M=0.66, S D=0.48]$, and unusual viewpoint $[M=0.54, S D=0.50]$ ) to the model did not account for significantly more variance in response accuracy than the null model, $\chi^{2}(3)=3.68, p=.298($ see Figure 3$)$.

\section{Experiment 4}




\section{RUNNING HEAD: MEMORY FOR WELL-KNOWN PLACES}

The odds of participants responding modified to modified and unmodified images were compared between young and older adults. A logistic mixed model was constructed with group (young, older), display type, and years lived in Toronto as fixed factors, and subject and landmark as random intercepts. The model showed a significant group by display type interaction, $b=-0.87, S E=0.4, Z=-2.16, p=.03$ (see Figure 4). Older adults' odds of responding that the image was modified were not significantly different for modified and unmodified trials, $b=0.01, S E=0.28, Z=0.02, p=.982, \mathrm{OR}=1.01,95 \% \mathrm{CI}[0.58,1.75]$. Younger adults were significantly less likely to respond that the image was modified for modified trials than for unmodified trials, $b=-0.87, S E=0.29, Z=-3.04, p=.002$, OR $=0.42,95 \% \mathrm{CI}[0.24,0.74]$. Older adults were significantly less likely than younger adults to say that the image was modified during unmodified trials, $b=-0.72, S E=0.36, Z=-2.00, p=.045$, OR $=0.49,95 \% \mathrm{CI}[0.24,0.98]$. During modified trials, there was no significant difference between the groups in their odds of responding that the image was modified, $b=-0.16, S E=0.37, Z=0.43, p=.665$, OR $=1.17$, 95\% CI $[0.57,2.41]$. Adding a group by modification type (size, transposition, feature) interaction for modified trials did not account for significantly more variance in the outcome variable, $\chi^{2}(2)=4.96, p=.084$. Lastly, there was a main effect of display type, such that participants were significantly more likely to respond that the image was modified on unmodified trials than on modified trials (see Table 1 and Figure 2c).

\section{Experiment 5}

A logistic mixed model was constructed with display type (modified, unmodified) and years lived in Toronto as fixed factors, and subject and landmark as random intercepts. Controlling for years lived in Toronto, participants' odds of responding that the image was modified were significantly lower for modified images than for unmodified images (Table 1; 
RUNNING HEAD: MEMORY FOR WELL-KNOWN PLACES

Figure 2d). Years lived in Toronto was not a significant predictor of participants' responses, $b=$ $0.02, S E=0.02, Z=0.65, p=.515, \mathrm{OR}=1.02,95 \% \mathrm{CI}[0.97,1.07]$. A likelihood ratio test showed that adding modification type (i.e., size $[M=0.28, S D=0.16]$, feature $[M=0.28, S D=$ 016], or transposition $[M=0.28, S D=0.16])$ to the model did not account for significantly more variance in the outcome variable, $\chi^{2}(2)=0.61, p=.738$.

\section{Eye tracking}

Correlations of the numeric variables, including years lived in Toronto, number of fixations, number of fixations in the interest area, and response accuracy are presented in Table 2. First, a mixed model was constructed with the number of fixations regressed on display type (i.e., modified vs. unmodified images), response accuracy, and their interaction, with subject and landmark as random intercepts. Controlling for the number of years lived in Toronto, we found a significant display type by accuracy interaction on the number of fixations, $\chi^{2}(1)=7.46, p=.006$ (Figure 4). Controlling for the number of years lived in Toronto, response accuracy was a significant predictor of the number of fixations for modified images, $b=0.86, S E=0.32$, $t(534.40)=2.70, p=.007$ (Table 3). For unmodified images, response accuracy was not a significant predictor of the number of fixations, $b=-0.40, S E=0.30, t(540.32)=-1.31, p=.192$. We then explored whether for modified images, modification type (i.e., size, transposition, and feature) predicted the number of fixations. A likelihood ratio test showed that adding modification type to the model did not account for significantly more variance in the number of fixations over and above the simpler model that included years lived in Toronto and display type as predictors, $\chi^{2}(2)=0.75, p=.686$.

We then built models with the same set of predictors, but this time focusing on the proportion of fixations in the interest areas as the outcome variable. Controlling for the number 
of years lived in Toronto and the size of the interest area, there was no significant display type by response accuracy interaction on the number of fixations in the interest areas (Figure 5), $\chi^{2}(1)=$ 0.402, $p=.895$. Controlling for the size of the interest area, the proportions of eye fixations in the interest areas were not significantly different between unmodified $(M=0.25, S D=0.23)$ and modified images $(M=0.27, S D=0.26), b=0.01, S E=0.02, t(356)=0.25, p=.802$. Adding the modification type by response accuracy interaction to the model did not explain significantly more variance in the number of fixations in the interest areas, $\chi^{2}(2)=3.03, p=.220$.

\section{Discussion}

To better understand the nature of long-term spatial memory representations, we used declarative memory tasks and eye tracking to examine whether visual properties inherent to landmarks would be less well-noticed than changes to the spatial locations of landmarks with respect to one another. Participants demonstrated poor accuracy across all experimental conditions in detecting even gross modifications to landmarks. This was true even when participants first imagined experiencing the route leading up to an unmodified or modified landmark (but not when the landmark presented was completely different or in an unexpected location). The nature of participants' poor performance was inconsistent across experiments, with modifications mistakenly detected in unmodified images in some experiments but not in others. Performance nevertheless remained poor in a forced-choice condition (Experiment 3). Although we expected that a more incidental measure, such as eye movements, would be more sensitive to these dramatic changes, the same pattern was reflected in the eye movement data, as significant differences only in the overall number of fixations to the scenes were found when participants were aware or believed, whether erroneously or correctly, that an image was modified in some way. Specifically, increased accuracy, and by extension conscious awareness, 
in identifying scene modification was positively related to global changes in visual exploration, but not to viewing that was specifically directed to the modification itself.

Here we found that changes to the visual appearance of landmarks are unlikely to be noticed, whereas changes to the locations of landmarks may be more detectable, but even those elements might not be detected if most elements within a scene are left intact. This pattern of findings resembles the classic demonstration that, despite everyday experience handling pennies, participants have trouble identifying the true penny among slightly modified versions, unless the difference is one of size and colour (Nickerson \& Adams, 1979). Why might this be so? In both instances, we may be conscious only of those visual changes that are essential to acting on the world to achieve a goal, whether it is navigating to a landmark or grasping the correct coin to purchase an item (Goodale, Milner, Jakobson, \& Carey, 1991). Visual details that are incidental to such goals might interfere with the task at hand, either by slowing down the organism or serving as an outright source of distraction. As a consequence, such details can go unnoticed even if they become task-relevant. This phenomenon may apply as well to landmarks transposed to a different context. For the purposes of navigation, it is the relational representation of multiple landmarks, with no single landmark particularly important. As long as the single landmark and/or its features fits unobtrusively into the configuration, it is unlikely to be noticed. If it becomes salient in its new location, then it becomes noticed, such as when the $\mathrm{CN}$ tower is transposed away from the waterfront and to the centre of a residential neighbourhood.

Until now, it was largely unknown what the effects of changes to perceptual elements of very familiar landmarks located in extended, real-world spaces that have been navigated extensively. The literature on newly encountered places, however, may have provided some clues. Earlier research in humans and non-human animals shows that the geometry of enclosed 
spaces is processed differently from, and may take precedence over, landmark identity in navigation. For example, it appears that rats and young children are naturally inclined to rely on enclosed geometry over salient environmental features (e.g., wall colour) as reorienting stimuli to navigate; only with training and experience do landmarks or identifying features become more prominent (Cheng \& Newcombe, 2005; Spelke \& Lee, 2012). This geometric bias is reflected in the response of the parahippocampal place area (PPA), a scene-sensitive brain region that codes visual properties of objects that signal their navigational relevance, including viewpoint changes (Epstein, Graham, \& Downing, 2003; Troiani et al., 2014), but only until the scene becomes more familiar to participants within the context of the experiment (Marchette, Ryan, \& Epstein, 2017). An example of visual processing in the service of navigation is the finding that, unlike objects, which are vulnerable to mirror-image confusion, scenes are discriminated from their mirror image by infant and adult humans and by animals (Cheng \& Newcombe, 2005; Lee \& Spelke, 2010). However, whereas activity in the retrosplenial cortex (RSC), which has also been implicated in spatial memory and navigation, is modulated by scene reversals, activity in the PPA appears to be unaffected (Dilks et al., 2011). This dissociation provides evidence that scene processing occurs along two visual streams, one for the purpose of navigation in the RSC, which is sensitive to visual image changes, and the other for categorizing scenes in the PPA, which is resilient to such changes (Dilks et al., 2011). Both regions are responsive to navigating in wellknown environments (Patai et al., 2019).

Research in hippocampally lesioned and aged rats and humans might provide additional insight. Rats with extensive prior experience in a complex 'village maze' appear to navigate based on the configuration of the room that the maze was in and not based on visual details (Winocur et al., 2010a,b). Similarly, memory-impaired and healthy older adults with many 
years' experience navigating in downtown Toronto can represent the spatial configuration of the locations occupied by landmarks and scenes that they had trouble recognizing and retrieving when imagining walking along well-known routes (Herdman et al., 2015; Rosenbaum et al., 2005 , 2012). Thus, even within representations of large-scale spaces, visual features of particular landmarks might not be retained by a system that is concerned with spatial navigation.

Our data converge on an idea introduced earlier that spatial memory representations of familiar environments are organized as schematic cognitive maps that preserve the gist of the environment without rich contextual details that may be necessary for re-experiencing it (Rosenbaum et al., 2001). In this sense, remote spatial memories are similar to remote semantic or context-free memories, which can be retrieved independently of the hippocampus. Therefore, with time and experience, very old spatial memories may become more gist-like in nature or common elements may be extracted to form schemas (Kan, Rosenbaum, \& Verfaellie, 2020). The current findings support predictions made by the Trace Transformation Theory (Winocur \& Moscovitch, 2011), such that as long as the overall spatial configuration of landmarks remains intact, even gross changes to landmarks can go unnoticed (Moscovitch et al., 2016). In contrast to previous studies of newly learned scenes (Ryan et al., 2000; Smith \& Squire, 2018), eye movements were not attracted to the modified region. Instead, global appreciation that a change had occurred predicted the overall manner of viewing, captured by the number of gaze fixations made to the scene in its entirety.

In sum, we show that changes to very familiar landmarks and scenes typically go unnoticed, particularly when the change does not appear to disrupt the balance of the landmark configuration needed for navigation. When participants believed that the scene had been modified in some way, as inferred from their performance in the experimental conditions, they 
engaged in greater visual exploration of the scene, independent of the precise modification. Our findings suggest that when navigating in extended, real-world spaces, what matters is the geometry of the environment but not the features of elements within it. 


\section{RUNNING HEAD: MEMORY FOR WELL-KNOWN PLACES}

\section{Author Contributions}

R.S.R., G.W., and M.M. developed the study concept. R.S.R., J.D.R., and M.M. contributed to the study design. Testing, data collection, and data analysis were performed by R.S.R., J.H., S.A., and M.D. All authors contributed to interpretation of the data. R.S.R., J.D.R., and M.M. drafted the paper, and J.H. provided critical input on revisions. All authors approved the final version of the paper for submission. 


\section{Acknowledgments}

This study was funded by a Canada First Research Excellence Fund (CFREF) Vision: Science to Applications (VISTA) York Research Chair and Natural Sciences and Engineering Research Council (NSERC) Grant RGPIN-04238-2015 to RSR and NSERC Grant A837 to MM. We thank Marie-Eve Couture, Doug McQuiggan, and Ryan Aloysius for technical assistance. 


\section{References}

Bates, D., Maechler, M., \& Bolker, B. (2012). lme4: Linear mixed-effects models using S4 classes (R Package). doi: http://cran.r-project.org/web/packages/lme4/index.html

Burgess N. (2008). Spatial cognition and the brain. Annals of the New York Academy of Sciences, 1124, 77-97. https://doi.org/10.1196/annals.1440.002

Cermak, L. S., \& O'Connor, M. (1983). The anterograde and retrograde retrieval ability of a patient with amnesia due to encephalitis. Neuropsychologia, 21(3), 213-234.

https://doi.org/10.1016/0028-3932(83)90039-8

Cheng, K., \& Newcombe, N. S. (2005). Is there a geometric module for spatial orientation? Squaring theory and evidence. Psychonomic bulletin \& review, 12(1), 1-23. https://doi.org/10.3758/bf03196346

Dilks, D. D., Julian, J. B., Kubilius, J., Spelke, E. S., \& Kanwisher, N. (2011). Mirror-image sensitivity and invariance in object and scene processing pathways. Journal of Neuroscience, 31, 11305-11312. https://doi.org/10.1523/JNEUROSCI.1935-11.2011

Eichenbaum H. (2017). The role of the hippocampus in navigation is memory. Journal of neurophysiology, 117(4), 1785-1796. https://doi.org/10.1152/jn.00005.2017

Ekstrom, A.D., Spiers, H.J., Bohbot, V.D., \& Rosenbaum, R.S. (2018). Human Spatial Navigation. Princeton University Press.

Epstein, R., Graham, K. S., \& Downing, P. E. (2003). Viewpoint-specific scene representations in human parahippocampal cortex. Neuron, 37(5), 865-876. https://doi.org/10.1016/s08966273(03)00117-X

Epstein, R. A., Patai, E. Z., Julian, J. B., \& Spiers, H. J. (2017). The cognitive map in humans: spatial navigation and beyond. Nature neuroscience, 20(11), 1504-1513. https://doi.org/10.1038/nn.4656

Gallistel, C.R. (1989). Animal cognition: the representation of space, time and number. Annual Review of Psychology, 40, 155-189. DOI: 10.1146/annurev.ps.40.020189.001103

Goodale, M. A., Milner, A. D., Jakobson, L. S., \& Carey, D. P. (1991). A neurological dissociation between perceiving objects and grasping them. Nature, 349(6305), 154-156. https://doi.org/10.1038/349154a0

Guderian, S., Dzieciol, A. M., Gadian, D. G., Jentschke, S., Doeller, C. F., Burgess, N., Mishkin, M., \& Vargha-Khadem, F. (2015). Hippocampal Volume Reduction in Humans Predicts Impaired Allocentric Spatial Memory in Virtual-Reality Navigation. Journal of Neuroscience, 35(42), 14123-14131. https://doi.org/10.1523/JNEUROSCI.0801-15.2015 
Hannula, D.E., Ryan, J.D., Warren, D.E. (2017). Beyond long-term declarative memory: Hippocampal contributions to perception, short-term retention, and unconscious memory expression. In Hannula, D.E. \& Duff, M. (Eds), The Hippocampus from Cells to Systems: Structure, Connectivity, and Functional Contributions to Memory and Flexible Cognition. pp. 281-336.

Hainmueller, T., \& Bartos, M. (2018). Parallel emergence of stable and dynamic memory engrams in the hippocampus. Nature, 558(7709), 292-296. https://doi.org/10.1038/s41586-0180191-2

Herdman, K. A., Calarco, N., Moscovitch, M., Hirshhorn, M., \& Rosenbaum, R. S. (2015). Impoverished descriptions of familiar routes in three cases of hippocampal/medial temporal lobe amnesia. Cortex; a journal devoted to the study of the nervous system and behavior, 71, 248263. https://doi.org/10.1016/j.cortex.2015.06.008

Hunsaker, M. R., \& Kesner, R. P. (2018). Unfolding the cognitive map: The role of hippocampal and extra-hippocampal substrates based on a systems analysis of spatial processing. Neurobiology of learning and memory, 147, 90-119. https://doi.org/10.1016/j.nlm.2017.11.012

Kan, I. P., Rosenbaum, R. S., \& Verfaellie, M. (2020). Schema processing across the lifespan: From theory to applications. Cognitive neuropsychology, 37(1-2), 1-7. https://doi.org/10.1080/02643294.2020.1736019

Lee, S. A., \& Spelke, E. S. (2010). Two systems of spatial representation underlying navigation. Experimental brain research, 206(2), 179-188. https://doi.org/10.1007/s00221-0102349-5

Lever, C., Burgess, N., Cacucci, F., Hartley, T., \& O'Keefe, J. (2002). What can the hippocampal representation of environmental geometry tell us about Hebbian learning? Biological cybernetics, 87(5-6), 356-372. https://doi.org/10.1007/s00422-002-0360-z

Manns, J. R., \& Eichenbaum, H. (2009). A cognitive map for object memory in the hippocampus. Learning \& memory (Cold Spring Harbor, N.Y.), 16(10), 616-624. https://doi.org/10.1101/lm.1484509

Marchette, S. A., Ryan, J. \& Epstein, R. A. (2017). Schematic representations of local environmental space guide goal-directed navigation. Cognition, 158, 68-80. doi:10.1016/j.cognition.2016.10.005 (2017).

Morris, R. G., Garrud, P., Rawlins, J. N., \& O'Keefe, J. (1982). Place navigation impaired in rats with hippocampal lesions. Nature, 297(5868), 681-683. https://doi.org/10.1038/297681a0

Moscovitch, M., Cabeza, R., Winocur, G., \& Nadel, L. (2016). Episodic Memory and Beyond: The Hippocampus and Neocortex in Transformation. Annual review of psychology, 67, 105-134. https://doi.org/10.1146/annurev-psych-113011-143733 
Moser, E. I., Moser, M. B., \& McNaughton, B. L. (2017). Spatial representation in the hippocampal formation: a history. Nature neuroscience, 20(11), 1448-1464. https://doi.org/10.1038/nn.4653

O'Keefe, J., \& Dostrovsky, J. (1971). The hippocampus as a spatial map. Preliminary evidence from unit activity in the freely-moving rat. Brain research, 34(1), 171-175. https://doi.org/10.1016/0006-8993(71)90358-1

O’Keefe, J., Nadel, L. (1978). The Hippocampus as a Cognitive Map. Oxford: Clarendon.

Patai, E. Z., Javadi, A. H., Ozubko, J. D., O'Callaghan, A., Ji, S., Robin, J., Grady, C., Winocur, G., Rosenbaum, R. S., Moscovitch, M., \& Spiers, H. J. (2019). Hippocampal and Retrosplenial Goal Distance Coding After Long-term Consolidation of a Real-World Environment. Cerebral cortex, 29(6), 2748-2758. https://doi.org/10.1093/cercor/bhz044

Quiroga, R. Q., Reddy, L., Kreiman, G., Koch, C., \& Fried, I. (2005). Invariant visual representation by single neurons in the human brain. Nature, 435(7045), 1102-1107. https://doi.org/10.1038/nature03687

Rosenbaum, R. S., Priselac, S., Köhler, S., Black, S. E., Gao, F., Nadel, L., \& Moscovitch, M. (2000). Remote spatial memory in an amnesic person with extensive bilateral hippocampal lesions. Nature neuroscience, 3(10), 1044-1048. https://doi.org/10.1038/79867

Rosenbaum, R. S., Winocur, G., \& Moscovitch, M. (2001). New views on old memories: reevaluating the role of the hippocampal complex. Behavioural brain research, 127(1-2), 183197. https://doi.org/10.1016/s0166-4328(01)00363-1

Rosenbaum, R. S., Gao, F., Richards, B., Black, S. E., \& Moscovitch, M. (2005). "Where to?" remote memory for spatial relations and landmark identity in former taxi drivers with Alzheimer's disease and encephalitis. Journal of cognitive neuroscience, 17(3), 446-462. https://doi.org/10.1162/0898929053279496

Rosenbaum, R. S., Winocur, G., Binns, M. A., \& Moscovitch, M. (2012). Remote spatial memory in aging: all is not lost. Frontiers in aging neuroscience, 4, 25. https://doi.org/10.3389/fnagi.2012.00025

Ryan, J. D., Althoff, R. R., Whitlow, S., \& Cohen, N. J. (2000). Amnesia is a deficit in relational memory. Psychological science, 11(6), 454-461. https://doi.org/10.1111/1467-9280.00288

Ryan, J. D., Shen, K., \& Liu, Z. X. (2020). The intersection between the oculomotor and hippocampal memory systems: empirical developments and clinical implications. Annals of the New York Academy of Sciences, 1464(1), 115-141. https://doi.org/10.1111/nyas.14256 
Scoville, W. B., \& Milner, B. (1957). Loss of recent memory after bilateral hippocampal lesions. Journal of neurology, neurosurgery, and psychiatry, 20(1), 11-21.

https://doi.org/10.1136/jnnp.20.1.11

Smith, C. N., Hopkins, R. O., \& Squire, L. R. (2006). Experience-dependent eye movements, awareness, and hippocampus-dependent memory. Journal of Neuroscience, 26(44), 1130411312. https://doi.org/10.1523/JNEUROSCI.3071-06.2006

Smith, C. N., \& Squire, L. R. (2018). Awareness of what is learned as a characteristic of hippocampus-dependent memory. Proceedings of the National Academy of Sciences of the United States of America, 115(47), 11947-11952. https://doi.org/10.1073/pnas.1814843115

Spelke, E. S., \& Lee, S. A. (2012). Core systems of geometry in animal minds. Philosophical transactions of the Royal Society of London. Series B, Biological sciences, 367(1603), 27842793. https://doi.org/10.1098/rstb.2012.0210

Squire, L. R., Genzel, L., Wixted, J. T., \& Morris, R. G. (2015). Memory consolidation. Cold Spring Harbor perspectives in biology, 7(8), a021766.

https://doi.org/10.1101/cshperspect.a021766

Teng, E., \& Squire, L. R. (1999). Memory for places learned long ago is intact after hippocampal damage. Nature, 400(6745), 675-677. https://doi.org/10.1038/23276

Troiani, V., Stigliani, A., Smith, M. E., \& Epstein, R. A. (2014). Multiple object properties drive scene-selective regions. Cerebral Cortex, 24(4), 883-897. https://doi.org/10.1093/cercor/bhs364

Winocur, G., \& Moscovitch, M. (2011). Memory transformation and systems consolidation. Journal of the International Neuropsychological Society, 17(5), 766-780. https://doi.org/10.1017/S1355617711000683

Winocur, G., Moscovitch, M., Rosenbaum, R. S., \& Sekeres, M. (2010a). A study of remote spatial memory in aged rats. Neurobiology of aging, 31(1), 143-150.

https://doi.org/10.1016/j.neurobiolaging.2008.03.016

Winocur, G., Moscovitch, M., Rosenbaum, R. S., \& Sekeres, M. (2010b). An investigation of the effects of hippocampal lesions in rats on pre- and postoperatively acquired spatial memory in a complex environment. Hippocampus, 20(12), 1350-1365. https://doi.org/10.1002/hipo.20721

Wright, D.B., Horry, R. \& Skagerberg, E.M. (2009). Functions for traditional and multilevel approaches to signal detection theory. Behavior Research Methods 41, 257-267.

https://doi.org/10.3758/BRM.41.2.257 
Table 1. Summary of main findings from behavioural experiments. The effect of display type on the odds of participants responding that the image was modified.

\begin{tabular}{ccccccccc}
\hline & \multicolumn{2}{c}{ Unmodified } & \multicolumn{2}{c}{ Modified } & Odds & 95\% CI & $Z$ & $p$ \\
\hline Experiment & $M$ & $S D$ & $M$ & $S D$ & Ratio & & & \\
\hline 1 & 0.33 & 0.07 & 0.47 & 0.09 & 1.75 & {$[1.34,2.29]$} & 4.13 & $<.001$ \\
2 & 0.28 & 0.12 & 0.40 & 0.14 & 1.89 & {$[0.84,4.26]$} & 1.53 & .126 \\
4 & 0.37 & 0.10 & 0.27 & 0.04 & 0.65 & {$[0.44,0.96]$} & -2.17 & .030 \\
5 & 0.36 & 0.17 & 0.28 & 0.16 & 0.67 & {$[0.45,0.98]$} & -2.09 & .037 \\
\hline
\end{tabular}


Table 2. Pearson zero-order correlations between years lived in Toronto, average eye-tracking measures, and average accuracy of responses across participants in Experiment 5.

\begin{tabular}{lllll}
\hline Variable & 1 & 2 & 3 & 4 \\
\hline 1. Years lived in Toronto & - & & & \\
2. Proportion of Fixations in the & 0.006 & - & & \\
$\quad$ Interest Areas & & & & \\
3. Number of Fixations & -0.249 & -0.392 & - & \\
4. Response Accuracy & -0.305 & -0.134 & 0.149 & - \\
\hline
\end{tabular}


Table 3. Overall number of eye fixations regressed on display type by accuracy interaction, controlling for years lived in Toronto in Experiment 5.

\begin{tabular}{lcccc}
\hline & \multicolumn{3}{c}{ Number of Fixations } \\
\cline { 2 - 4 } Fixed Effects & $b$ & $S E$ & $d f$ & $t$ \\
\hline Intercept & 23.32 & 1.06 & 23.98 & $22.03^{* * *}$ \\
Years Lived in Toronto & -0.09 & 0.07 & 22.01 & -1.32 \\
Unmodified & 0.41 & 0.29 & 534.28 & 1.40 \\
Accuracy & 0.86 & 0.32 & 534.40 & $2.70^{* *}$ \\
Image Modification $\times$ Accuracy & -1.26 & 0.46 & 538.96 & $-2.74^{* *}$ \\
\hline Random Effects & $b$ & $S D$ & & \\
\hline Subject (Intercept error variance) & 8.58 & 2.93 & & \\
Landmark (Intercept error variance) & 0.75 & 0.86 & & \\
Residual & 5.17 & 2.27 & & \\
\hline
\end{tabular}

Note. Modified condition was used as a reference category in the analysis.

$* p<.05, * * p<.01, * * * p<.001$ 


\section{Figure Captions}

Figure 1. Photograph of the CN Tower and Rogers Centre, located in downtown Toronto. The $\mathrm{CN}$ Tower appears smaller in the photo than its actual size.

Figure 2. Probability of responding "modified" by display type in Experiments 1 (A), 2 (B), 4 (C), and 5 (D). The boxes inside the violin plots encompass interquartile ranges and the error bars represent standard errors.

Figure 3. Probability of correct response by modification type in Experiment 3. Participants did not show significant differences in response accuracy across the modification conditions.

Figure 4. Probability of participants responding that the image is modified across unmodified and modified trials in younger and older adults in Experiment 4. The graph shows a significant display type by group interaction.

Figure 5. Number of fixations by image modification and response accuracy in Experiment 5. The error bars indicate standard errors.

Figure 6. Proportion of fixations in the interest areas by image modification and response accuracy in Experiment 5. The error bars indicate standard errors. 
RUNNING HEAD: MEMORY FOR WELL-KNOWN PLACES

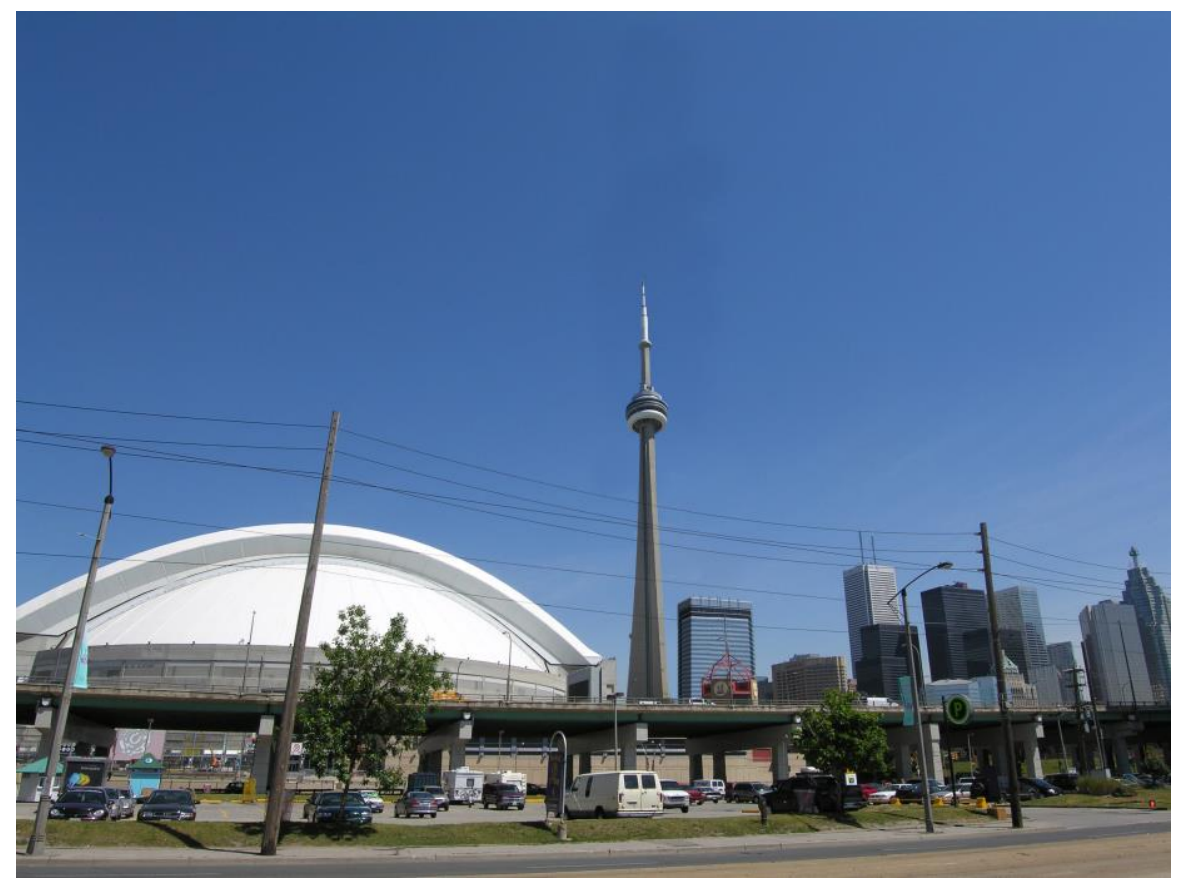


A

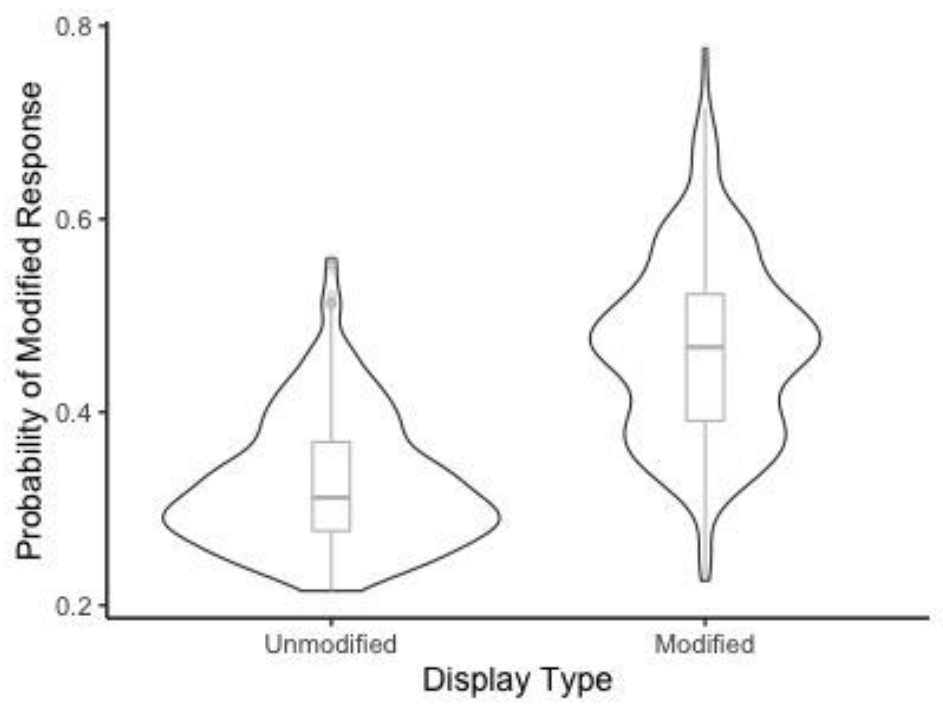

C

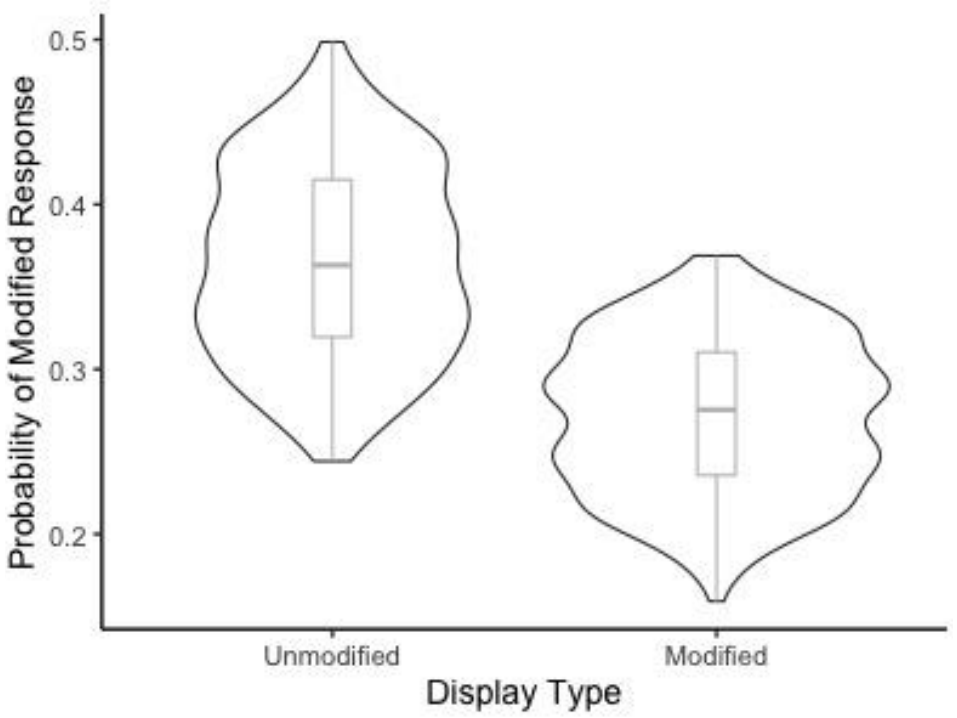

B

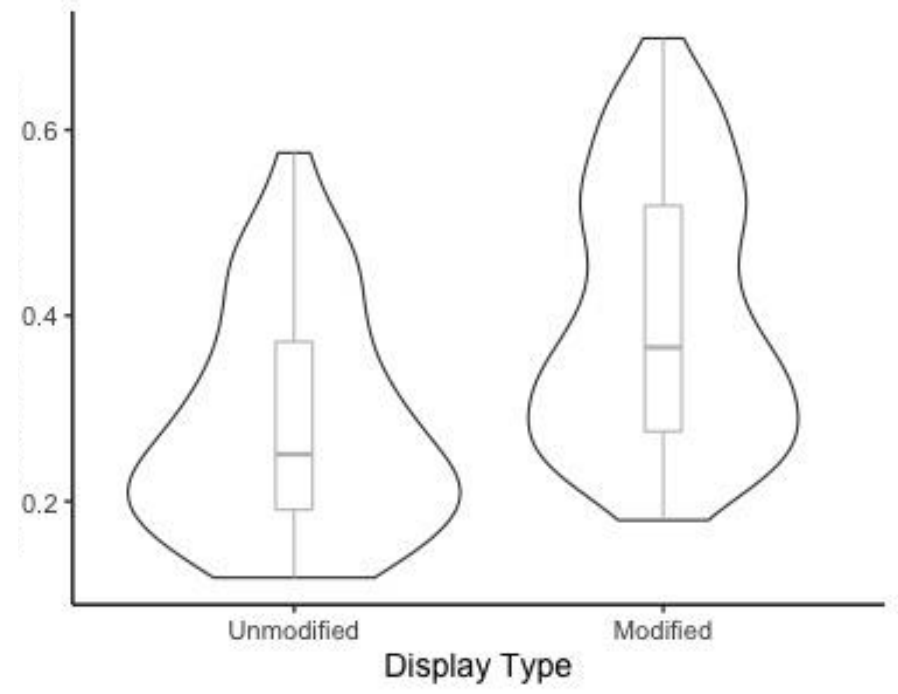

D

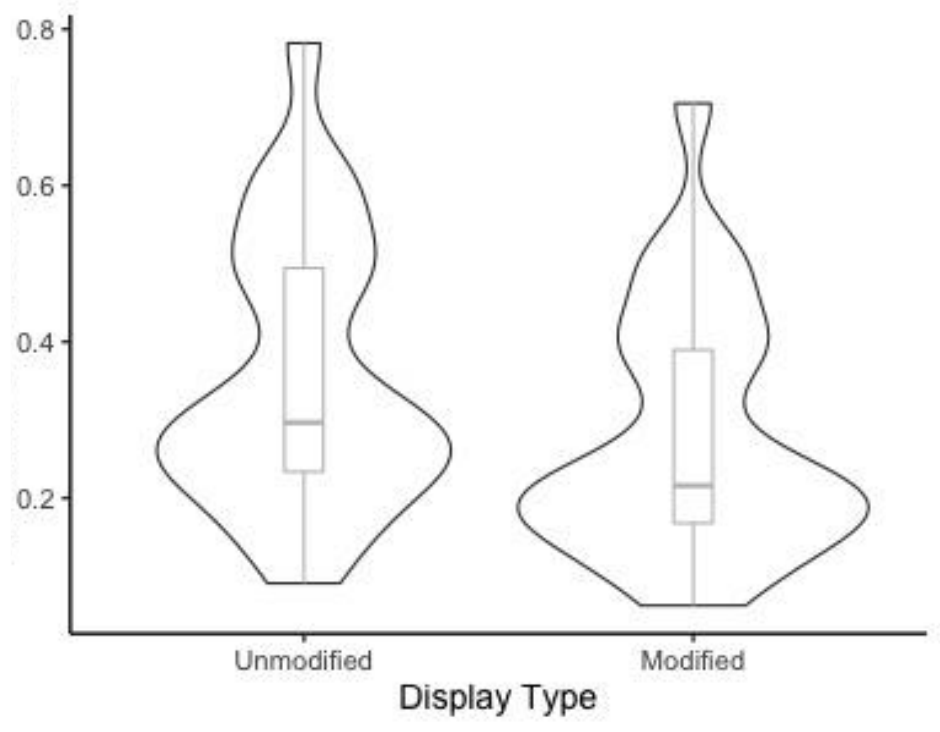


RUNNING HEAD: MEMORY FOR WELL-KNOWN PLACES

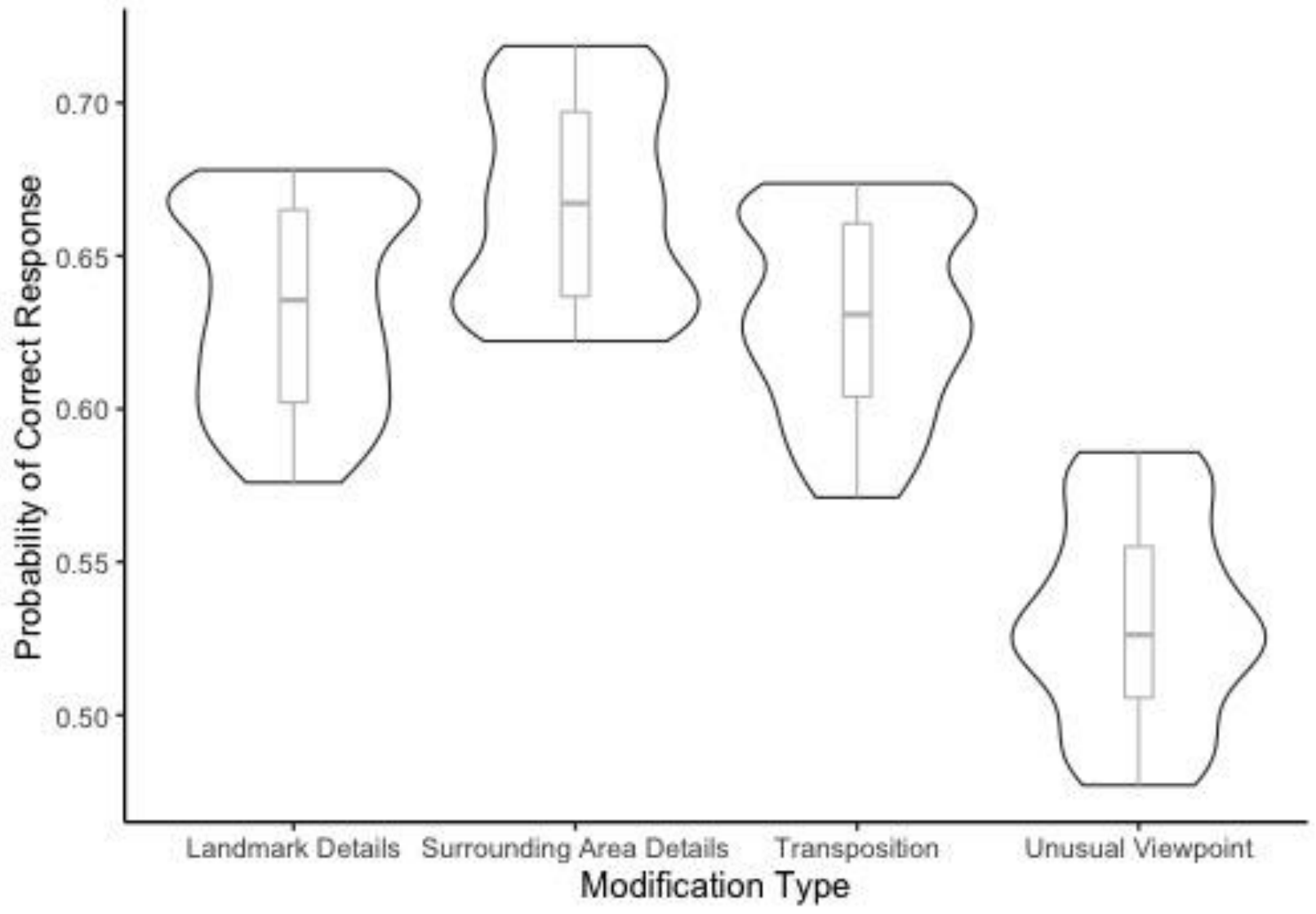


RUNNING HEAD: MEMORY FOR WELL-KNOWN PLACES

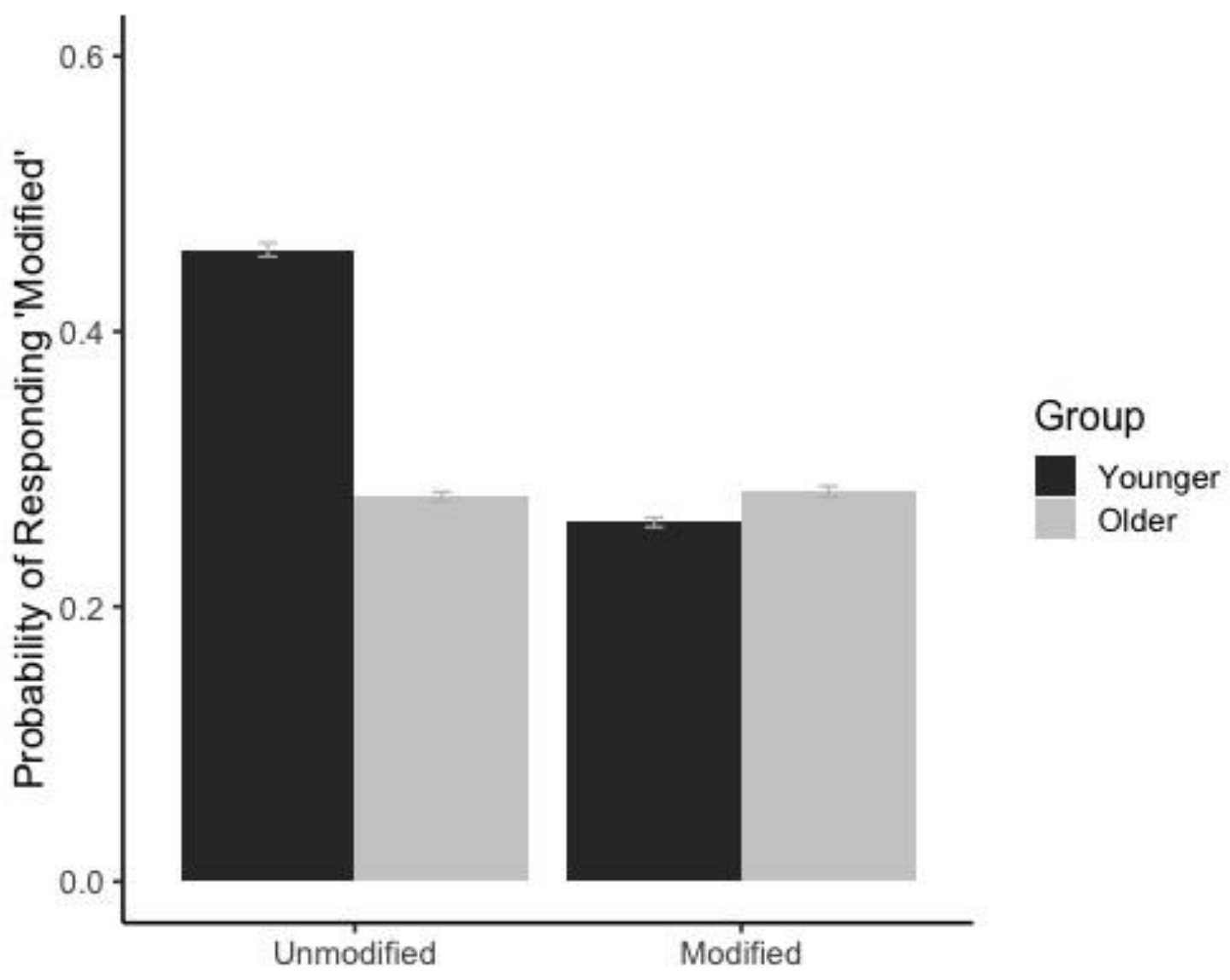


RUNNING HEAD: MEMORY FOR WELL-KNOWN PLACES

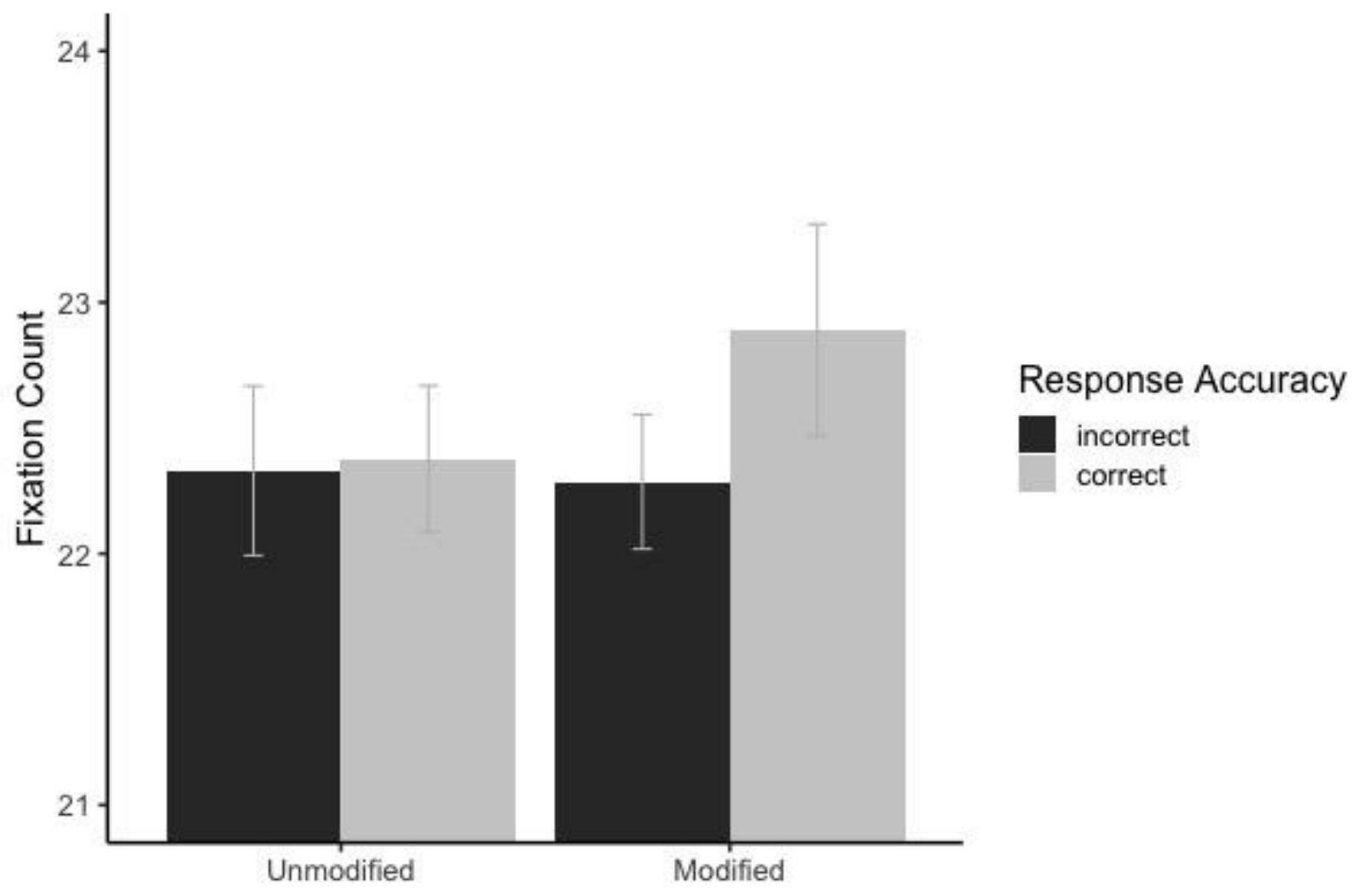


RUNNING HEAD: MEMORY FOR WELL-KNOWN PLACES

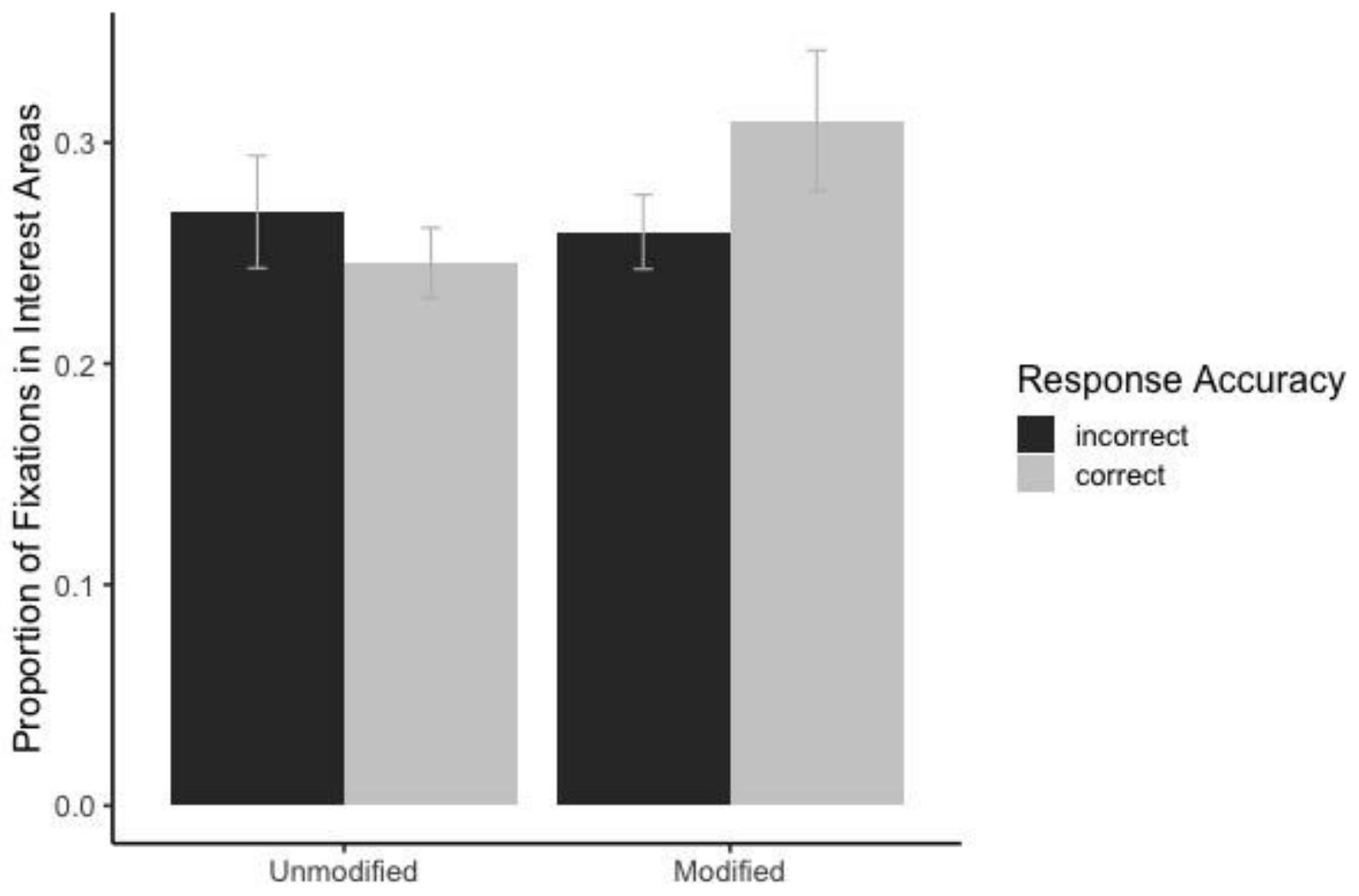

